\title{
Summation of Multiple Fourier Series in Matrix Weighted $L_{p}$-Spaces
}

\begin{abstract}
Morten Nielsen
Department of Mathematical Sciences, Aalborg University, Fredrik Bajers Vej 7G, 9220 Aalborg East, Denmark
\end{abstract}

Correspondence should be addressed to Morten Nielsen; mnielsen@math.aau.dk

Received 28 January 2013; Accepted 16 February 2013

Academic Editor: Baoding Liu

Copyright (C) 2013 Morten Nielsen. This is an open access article distributed under the Creative Commons Attribution License, which permits unrestricted use, distribution, and reproduction in any medium, provided the original work is properly cited.

This paper is concerned with rectangular summation of multiple Fourier series in matrix weighted $L_{p}$-spaces. We introduce a product Muckenhoupt $A_{p}$ condition for matrix weights $W$ and prove that rectangular Fourier partial sums converge in the corresponding matrix weighted space $L_{p}\left(\mathbb{T}^{d} ; W\right), 1<p<\infty$, if and only if the weight satisfies the product Muckenhoupt $A_{p}$ condition. The same result is shown to hold true for other summation methods such as Cesàro and summation with the Jackson kernel.

\section{Introduction}

Let $\mathscr{M}$ be the family of nonnegative-definite $m \times m$ complexvalued matrices. A (periodic) matrix weight is by definition an integrable map $W: \mathbb{T}^{d} \rightarrow \mathscr{M}$. For a measurable vectorvalued function $\mathbf{f}=\left(f_{1}, \ldots, f_{m}\right)^{T}: \mathbb{T}^{d} \rightarrow \mathbb{C}^{m}$, let

$$
\|f\|_{L_{p}\left(\mathbb{T}^{d} ; W\right)}:=\left(\int_{\mathbb{T}^{d}}\left|W^{1 / p}(t) \mathbf{f}(t)\right|^{p} d t\right)^{1 / p}, \quad 1 \leq p<\infty,
$$

where $|\cdot|$ denotes the usual norm on $\mathbb{C}^{m}$. We let $L_{p}\left(\mathbb{T}^{d} ; W\right)$ denote the family $\left\{\mathbf{f}: \mathbb{T}^{d} \rightarrow \mathbb{C}^{m}:\|\mathbf{f}\|_{L_{p}\left(\mathbb{T}^{d} ; W\right)}<\infty\right\}$, and $L_{p}\left(\mathbb{T}^{d} ; W\right)$ becomes a Banach space when we factorize over $\mathscr{N}=\left\{\mathbf{f}:\|\mathbf{f}\|_{L_{p}\left(\mathbb{T}^{d} ; W\right)}=0\right\}$.

In this paper we are interested in convergence properties of multiple trigonometric series in $L_{p}\left(\mathbb{T}^{d} ; W\right)$ and how specific convergence properties of trigonometric series can be related to properties of the weight $W$. To be more specific, let $D_{n}(t)=\sum_{|k| \leq n} e^{-2 \pi i k t}$ denote the univariate Dirichlet kernel, and for $\mathbf{N} \in \mathbb{N}^{d}$ we define the rectangular kernel $D_{\mathbf{N}}(t):=$ $\prod_{j=1}^{d} D_{N_{j}}\left(t_{j}\right)$. Then

$$
S_{\mathbf{N}} f:=f * D_{\mathbf{N}}:=\int_{\mathbb{T}^{d}} f(t) D_{\mathbf{N}}(\cdot-t) d t, \quad f \in L_{1}\left(\mathbb{T}^{d}\right),
$$

defines the rectangular partial sum operator for the trigonometric system. We define the action of $S_{\mathrm{N}}$ on vector-valued functions $\mathbf{f}$ by letting it act separately on each coordinate function; that is,

$$
\left[S_{\mathbf{N}}(\mathbf{f})\right]_{j}=S_{\mathbf{N}}\left(f_{j}\right), \quad j=1,2, \ldots m .
$$

It is well known (see, e.g., [1, Theorem 3.5.7]) that $\| f-$ $S_{\mathbf{N}}(f) \|_{L_{p}\left(\mathbb{T}^{d}\right)} \rightarrow 0$, as $\min _{j} N_{j} \rightarrow+\infty$, for $1<p<$ $\infty$. An immediate corollary is that we have convergence of the partial sums $S_{\mathrm{N}}(\mathbf{f})$, in $L_{p}\left(\mathbb{T}^{d} ; \mathrm{Id}\right)$, for $1<p<\infty$ in the vector-valued case. However, it is not obvious what can be said about convergence of $S_{\mathbf{N}}(\mathbf{f})$ in $L_{p}\left(\mathbb{T}^{d} ; W\right)$ for a general matrix weight $W$. The main result of the preset paper completely characterizes the special class of weights that allow convergence; $S_{\mathbf{N}}(\mathbf{f})$ converges if and only if the weight $W$ satisfies a certain matrix Muckenhoupt $A_{p}$ product condition. Moreover, the characterization relies solely on certain localization properties of the Dirichlet kernels shared by many other summation kernels. So, in addition, we prove that the rectangular Cesàro means and approximation using the Jackson kernels converge in $L_{p}\left(\mathbb{T}^{d} ; W\right)$ if and only if the weight $W$ satisfies the mentioned matrix Muckenhoupt $A_{p}$ product condition.

At a first glance, the study of vector-valued operators like $S_{\mathrm{N}}$ on $L_{p}\left(\mathbb{T}^{d} ; W\right)$ may seem artificial, but let us mention 
one important application to finitely generated shift invariant spaces where such mappings appear naturally. For a finite set of functions $\left\{f_{k}\right\}_{k=1}^{m}$ in $L_{2}\left(\mathbb{R}^{d}\right)$, the associated shift invariant space $S$ is given by

$$
S:=\overline{\operatorname{Span}\left\{f_{k}(\cdot-l): l \in \mathbb{Z}^{d}, k=1,2, \ldots, m\right\}} \subset L_{2}\left(\mathbb{R}^{d}\right) .
$$

A natural question to pose is whether $B:=\operatorname{Span}\left\{f_{k}(\cdot-l): l \in\right.$ $\left.\mathbb{Z}^{d}, k=1,2, \ldots, m\right\}$ forms some sort of "stable" generating system for $S$. Here stable can mean a Schauder basis, or even some weaker notion such as a block Schauder basis. Consider the vector valued system $\mathscr{T}:=\left\{\mathbf{e}_{j} e^{2 \pi i k \cdot t}: k \in \mathbb{Z}^{d}, j=\right.$ $1, \ldots, m\}$, where $\left\{\mathbf{e}_{j}\right\}_{j=1}^{m}$ is the standard basis for $\mathbb{C}^{m}$, and let $G$ the $m \times m$ Gram matrix be given by

$$
G_{i, j}:=\sum_{k \in \mathbb{Z}^{d}} \widehat{f}_{i}(\cdot-k) \overline{\widehat{f}_{j}(\cdot-k)},
$$

where the Fourier transform is given by $\hat{f}(\xi)$ := $\int_{\mathbb{R}^{d}} f(t) e^{-2 \pi i t \cdot \xi} d t$. One can show (see [2]) that the map $U: L_{2}\left(\mathbb{T}^{d} ; G\right) \rightarrow S$, given by

$$
U(\tau):=\left(\sum_{\ell=1}^{N} \tau_{\ell}(\xi) \widehat{\psi_{\ell}}\right)^{\vee}
$$

is an isometric isomorphism between $L_{2}\left(\mathbb{T}^{d} ; G\right)$ and $S$, satisfying $U\left(\mathbf{e}_{j} e^{-2 \pi i k \cdot t}\right)=f_{j}(\cdot-k)$. Hence, the metric properties of $B$ in $S$ are equivalent to the properties of $\mathscr{T}$ in $L_{2}\left(\mathbb{T}^{d} ; G\right)$. For example, $U$ will map $\left\{S_{\mathrm{N}}\right\}$ to a corresponding rectangular partial sum operators for $B$ on $S$. The same correspondence holds true for the other summation methods related to $\mathscr{T}$ such as rectangular Cesàro means and approximation using the Jackson kernels converge in $L_{p}\left(\mathbb{T}^{d} ; W\right)$ that will be discussed below.

It was proved by the present author [2] that the rectangular partial sums $\left\{U S_{\mathrm{N}}\right\}$ converge in $S$ precisely when $\left\{S_{\mathrm{N}}\right\}$ are uniformly bounded on $L_{2}(\mathbb{T} ; G)$ which happens exactly when $G$ is a Muckenhoupt $A_{2}$ product matrix weight.

The structure of this paper is as follows. In Section 2 we introduce a product $A_{p}$ condition for matrix weights. Then necessary and sufficient conditions for a convolution operator of product type (such as $S_{\mathbf{N}}$ ) to be bounded on $L_{p}\left(\mathbb{T}^{d} ; W\right)$ are given. Section 3 contains applications of the results in Section 2 to convolution operators induced by rectangular Dirichlet, Fejér, and Jackson kernels.

\section{The Muckenhoupt Condition and Operators on $L_{p}\left(\mathbb{T}^{d} ; W\right)$}

In this section we introduce a matrix Muckenhoupt $A_{p}$ product condition suitable for dealing with convolution operators of product type such as the partial sum operator $S_{\mathrm{N}}$ defined by (3). A sufficient condition for convolution operators of product type bounded on $L_{p}\left(\mathbb{T}^{d} ; W\right)$ is given in Proposition 5, while a converse type result is considered in Proposition 7. We prove that convolution operators with "nicely localized" kernels can only be uniformly bounded on $L_{p}\left(\mathbb{T}^{d} ; W\right)$ when $W$ satisfies the product $A_{p}$ condition.

The scalar $A_{p}$ condition was introduced by Muckenhoupt [3], and it was proved by Hunt et al. in their seminal paper [4] that the $A_{p}$ condition on a weight $w$ is necessary and sufficient for the Hilbert transform to be bounded on the weighted space $L_{p}(\mathbb{\nabla} ; w)$.

More recently, Hunt-Muckenhoupt-Wheeden type results for matrix weights have been considered. The matrix $A_{p}$ condition was introduced by Nazarov et al. [5-7] and they showed that it is the right condition for "standard" singular integral operators to be bounded on $L_{p}\left(\mathbb{T}^{d} ; W\right)$. The $A_{p}$ condition $(1<p<\infty)$ for weights $W: \mathbb{R}^{d} \rightarrow \mathscr{M}$ was originally stated in terms of dual matrices and average, but it was shown by Roudenko [8] to be equivalent to

$$
\sup _{B}\left(\int_{B}\left(\int_{B}\left\|W^{1 / p}(x) W^{-1 / p}(y)\right\|^{p^{\prime}} \frac{d y}{|B|}\right)^{p / p^{\prime}} \frac{d x}{|B|}\right)^{p^{\prime} / p},
$$

where the sup is taken over all open balls in $\mathbb{R}^{d}$ and $p^{\prime}=$ $p /(p-1)$ is the conjugate exponent.

Since our goal is to study operators of product type related to rectangular trigonometric partial sums, the condition given by (7) is not the appropriate one. The periodic weights satisfying (7) are well behaved when it comes to the study of square or spherical partial sum operators for trigonometric series. Let us therefore introduce a new and slightly modified Muckenhoupt condition. Inspired by $(7)$, we let $\mathscr{R}(d)$ denote the family of all rectangles in $\mathbb{R}^{d}$ of the form $R=I_{1} \times I_{2} \times$ $\cdots \times I_{d}$, with $I_{j}$ being a bounded open interval in $\mathbb{R}$. Then we consider the following more restrictive subclass of matrix weights.

Definition 1. Let $W: \mathbb{T}^{d} \rightarrow \mathscr{M}$ be a periodic matrix weight. For $1<p<\infty$, let $p^{\prime}=p /(p-1)$ denote the conjugate exponent to $p$. We say that $W$ belongs to the matrix Muckenhoupt (product) class $P A_{p}$ provided there exists a uniform constant $c_{W}$ such that

$$
\begin{aligned}
& A_{W}(R, p) \\
& \quad:=\int_{R}\left(\int_{R}\left\|W^{1 / p}(x) W^{-1 / p}(y)\right\|^{p^{\prime}} \frac{d y}{|R|}\right)^{p / p^{\prime}} \frac{d x}{|R|} \leq c_{W},
\end{aligned}
$$

for any $R \in \mathscr{R}(d)$.

Remark 2. For $d=1$, Definition 1 reduces to the standard matrix $A_{p}$ condition on $\mathbb{T}$, which we denote by $A_{p}(\mathbb{T})$. In the scalar case (i.e., $m=1$ ), Definition 1 reduces to the known product $A_{p}$ condition for scalar weights, which has a long history; see [9] and references therein.

The similarity of conditions (8) and (7) implies that many results for matrix $A_{p}$ weights have straightforward analogs in the product case; the proofs can be "translated verbatim." Let us state the following lemma which will be needed below. 
Lemma 3. Let $W: \mathbb{T}^{d} \rightarrow \mathscr{M}$ be a matrix weight. Then the following statements are equivalent for $1<p<\infty$ :

(i) $W \in P A_{p}$;

(ii) $W^{-p^{\prime} / p} \in P A_{p^{\prime}}$;

(iii) $\int_{R}\left(\int_{R}\left\|W^{1 / p}(x) W^{-1 / p}(y)\right\|^{p}(d x /|R|)\right)^{p^{\prime} / p}(d y /|R|) \quad \leq$ $c_{W}$, for all $R \in \mathscr{R}(d)$.

We refer the reader to Roudenko [8] for the proof of Lemma 3 in the nonproduct case.

The following Lemma reveals why one can expect $A P_{p}$ to be useful for product operators. A weight in $A P_{p}$ is uniformly in $A_{p}(\mathbb{T})$ in each of its $d$ variables.

Lemma 4. Let $W: \mathbb{T}^{d} \rightarrow M$ be a matrix weight, and let $1<p<\infty$. Then the following holds.

(a) For any rectangles $R \subseteq \widetilde{R} \subset \mathscr{R}(d)$,

$$
A_{W}(R, p) \leq\left(\frac{|\widetilde{R}|}{|R|}\right)^{p} A_{W}(\widetilde{R}, p) .
$$

(b) Suppose $W \in P A_{p}$; then the univariate weight $\xi_{j} \rightarrow$ $W(\xi)$, obtained by fixing the variables $\xi_{k}, k \neq j$, is uniformly in $\mathbb{A}_{p}(\mathbb{T})$ for a.e. $\left(\xi_{1}, \ldots, \xi_{j-1}, \xi_{j+1}, \ldots, \xi_{d}\right) \in$ $\mathbb{T}^{d-1}$.

Proof. For (a), we notice that whenever $R \subseteq \widetilde{R} \subset \mathscr{R}(d)$,

$$
\begin{aligned}
& A_{W}(R, p) \\
& \quad=\int_{R}\left(\int_{R}\left\|W^{1 / p}(x) W^{-1 / p}(t)\right\|^{p^{\prime}} \frac{d t}{|R|}\right)^{p / p^{\prime}} \frac{d x}{|R|} \\
& \quad \leq\left(\frac{|\widetilde{R}|}{|R|}\right)^{p} \int_{\widetilde{R}}\left(\int_{\widetilde{R}}\left\|W^{1 / p}(x) W^{-1 / p}(t)\right\|^{p^{\prime}} \frac{d t}{|\widetilde{R}|}\right)^{p / p^{\prime}} \frac{d x}{|\widetilde{R}|} \\
& \quad=\left(\frac{|\widetilde{R}|}{|R|}\right)^{p} A_{W}(\widetilde{R}, p) .
\end{aligned}
$$

Now we turn to the proof of (b). It suffices to consider $\widetilde{W}(t):=$ $W\left(t, \xi_{2}, \ldots, \xi_{d}\right)$ for $\left(\xi_{2}, \ldots, \xi_{d}\right) \in \mathbb{T}^{d-1}$ fixed. Given an interval $I \subset \mathbb{R}$, we form $R_{\varepsilon}=I_{\varepsilon}\left(\xi_{2}\right) \times \cdots \times I_{\varepsilon}\left(\xi_{d}\right)$, where $I_{\varepsilon}\left(\xi_{j}\right)$ is an interval of length $2 \varepsilon$ centered at $\xi_{j}$. First suppose $p \leq p^{\prime}$. Since
$W \in P A_{p}$, there exists a constant $c_{W}$ independent of $I \times R_{\varepsilon}$ such that

$$
\begin{gathered}
\frac{1}{\left|R_{\varepsilon}\right|^{2}} \int_{R_{\varepsilon}} \int_{R_{\varepsilon}}\left[\int _ { I } \left(\int_{I} \| W^{1 / p}(t, \mathbf{u}) W^{-1 / p}\right.\right. \\
\left.\left.\times(w, \mathbf{v}) \|^{p^{\prime}} \frac{d w}{|I|}\right)^{p / p^{\prime}} \frac{d t}{|I|}\right] d \mathbf{u} d \mathbf{v} \\
\leq \int_{R_{\varepsilon}} \int_{I}\left(\int_{R_{\varepsilon}} \int_{I}\left\|W^{1 / p}(t, \mathbf{u}) W^{-1 / p}(w, \mathbf{v})\right\|^{p^{\prime}}\right. \\
\left.\times \frac{d w d \mathbf{v}}{|I| \cdot\left|R_{\varepsilon}\right|}\right)^{p / p^{\prime}} \frac{d t d \mathbf{u}}{|I| \cdot\left|R_{\varepsilon}\right|} \\
=A_{W}(R, p) \leq c_{W},
\end{gathered}
$$

where we have used the continuous embedding $L_{1}\left(R_{\varepsilon} ; d \mathbf{v} /\left|R_{\varepsilon}\right|\right) \hookrightarrow L_{p / p^{\prime}}\left(R_{\varepsilon} ; d \mathbf{v} /\left|R_{\varepsilon}\right|\right)$. Hence, by Lebesgue's differentiation theorem, for almost every $\left(\xi_{2}, \ldots, \xi_{d}\right) \in \mathbb{T}^{d-1}$,

$$
\begin{aligned}
c_{W} & \geq \lim _{\varepsilon \rightarrow 0^{+}} A_{W}\left(I \times R_{\varepsilon}, p\right) \\
& =\int_{I}\left(\int_{I}\left\|\widetilde{W}^{1 / p}(t) \widetilde{W}^{-1 / p}(w)\right\|^{p^{\prime}} \frac{d w}{|I|}\right)^{p / p^{\prime}} \frac{d t}{|I|} \\
& =A_{\widetilde{W}}(I, p),
\end{aligned}
$$

where the constant is independent of $I$ and $\left(\xi_{2}, \ldots, \xi_{d}\right)$. It follows that $\widetilde{W}$ is uniformly in $\mathbb{A}_{p}(\mathbb{T})$ for a.e. $\left(\xi_{2}, \ldots, \xi_{d}\right) \in$ $\mathbb{T}^{d-1}$. In the case $p^{\prime}<p$, we use Lemma 3 to conclude that $W^{-p^{\prime} / p} \in P A_{p^{\prime}}$, which implies the following estimate:

$$
\begin{gathered}
\int_{R_{\varepsilon}} \int_{I}\left(\int_{R_{\varepsilon}} \int_{I}\left\|W^{1 / p}(t, \mathbf{u}) W^{-1 / p}(w, \mathbf{v})\right\|^{p}\right. \\
\left.\times \frac{d t d \mathbf{u}}{|I| \cdot\left|R_{\varepsilon}\right|}\right)^{p^{\prime} / p} \frac{d w d \mathbf{v}}{|I| \cdot\left|R_{\varepsilon}\right|} \leq c_{W} .
\end{gathered}
$$

By repeating the argument from the $p \leq p^{\prime}$ case, we conclude that $\widetilde{W}^{-p^{\prime} / p}$ is uniformly in $A_{p^{\prime}}(\mathbb{T})$ which again by Lemma 3 implies that $\widetilde{W}$ is uniformly in $A_{p}(\mathbb{T})$.

We can now prove the following result that explains how to get from a bounded convolution operator on $L_{p}(\mathbb{T} ; W)$ to a bounded convolution operator on $L_{p}\left(\mathbb{T}^{d} ; W\right)$, for $W \in A P_{p}$, simply by forming the natural product kernel.

Proposition 5. Suppose that $\left\{K_{N}\right\}_{N \geq 0}$ is a sequence of convolution kernels defined on $\mathbb{T}$ for which the corresponding operators

$$
T_{N} f:=\int_{\mathbb{T}} f(t) K_{N}(\cdot-t) d t
$$


are uniformly bounded on $L_{p}(\mathbb{T} ; W)$ whenever $W \in A_{p}(\mathbb{T})$. Then the associated product convolution kernels

$$
\begin{array}{r}
K_{\mathbf{N}}(\xi)=\prod_{j=1}^{d} K_{N_{j}}\left(\xi_{j}\right), \quad \mathbf{N}=\left(N_{1}, \ldots, N_{d}\right) \in \mathbb{N}_{0}^{d}, \\
\xi \in \mathbb{T}^{d},
\end{array}
$$

induce a uniformly bounded family of operators on $L_{p}\left(\mathbb{T}^{d} ; W\right)$ for $W \in P A_{p}$.

Proof. Suppose that $W \in P A_{p}$. In the case $d=1$, there is nothing to prove. We focus on the case $d=2$; the reader can easily verify that the argument below generalizes to any $d \geq 3$.

According to Lemma $4(\mathrm{~b}), W_{\xi_{1}}:=W\left(\xi_{1}, \cdot\right)$ and $W_{\xi_{2}}:=$ $W\left(\cdot, \xi_{2}\right)$ satisfy uniform Muckenhoupt $A_{p}$-conditions a.e. on $\mathbb{T}$. Pick any $f \in L_{p}\left(\mathbb{T}^{2}, W\right)$. By Fubini's theorem, $f_{\xi_{1}}:=$ $f\left(\xi_{1}, \cdot\right) \in L_{p}\left(\mathbb{T}, W_{\xi_{1}}\right)$ and $f_{\xi_{2}}:=f\left(\cdot, \xi_{2}\right) \in L_{p}\left(\mathbb{T}, W_{\xi_{2}}\right)$ for a.e. $\left[\xi_{1}\right]$ and $\left[\xi_{2}\right]$, respectively.

We define

$$
\begin{aligned}
& T_{N}^{1} f:=K_{N} * f_{\xi_{2}}:=\int_{\mathbb{T}} f_{\xi_{2}}(t) K_{N}(\cdot-t) d t \\
& T_{M}^{2} f:=K_{M} * f_{\xi_{1}}:=\int_{\mathbb{T}} f_{\xi_{1}}(t) K_{M}(\cdot-t) d t .
\end{aligned}
$$

Notice that $T_{N, M} f=T_{N}^{1} T_{M}^{2} f$. By assumption,

$$
\begin{aligned}
& \int_{\mathbb{T}}\left|W_{\xi_{1}}^{1 / p}\left(\xi_{2}\right) T_{M}^{2} f_{\xi_{1}}\left(\xi_{2}\right)\right|^{p} d \xi_{2} \\
& \quad \leq C \int_{\mathbb{T}}\left|W_{\xi_{1}}^{1 / p}\left(\xi_{2}\right) f_{\xi_{1}}\left(\xi_{2}\right)\right|^{p} d \xi_{2}, \quad \text { a.e. }\left[\xi_{1}\right] .
\end{aligned}
$$

An integration yields

$$
\begin{aligned}
\int_{\mathbb{T}} \int_{\mathbb{T}}\left|W^{1 / p}\left(\xi_{1}, \xi_{2}\right) T_{M}^{2} f\left(\xi_{1}, \xi_{2}\right)\right|^{p} d \xi_{2} d \xi_{1} \\
\quad \leq C \int_{\mathbb{T}} \int_{\mathbb{T}}\left|W^{1 / p}\left(\xi_{1}, \xi_{2}\right) f\left(\xi_{1}, \xi_{2}\right)\right|^{p} d \xi_{2} d \xi_{1} .
\end{aligned}
$$

Similarly,

$$
\begin{aligned}
& \left\|T_{N, M} f\right\|_{L_{p\left(\mathbb{T}^{2}, W\right)}^{p}}^{p} \\
& \quad=\int_{\mathbb{T}} \int_{\mathbb{T}}\left|W^{1 / p}\left(\xi_{1}, \xi_{2}\right) T_{N}^{1} T_{M}^{2} f\left(\xi_{1}, \xi_{2}\right)\right|^{p} d \xi_{1} d \xi_{2} \\
& \leq C \int_{\mathbb{T}} \int_{\mathbb{T}}\left|W^{1 / p}\left(\xi_{1}, \xi_{2}\right) T_{M}^{2} f\left(\xi_{1}, \xi_{2}\right)\right|^{p} d \xi_{1} d \xi_{2} \\
& \leq C^{2} \int_{\mathbb{T}} \int_{\mathbb{T}}\left|W^{1 / p}\left(\xi_{1}, \xi_{2}\right) f\right|^{p} d \xi_{1} d \xi_{2} .
\end{aligned}
$$

It follows that the family $\left\{T_{\mathbf{N}}\right\}_{\mathbf{N} \in \mathbb{N}_{0}^{2}}$ is uniformly bounded on $L_{p}\left(\mathbb{T}^{2} ; W\right)$.

We now turn to a converse type result to Proposition 5. Proposition 7 will show that well-localized trigonometric convolution kernels of product type can only be uniformly bounded on $L_{p}\left(\mathbb{T}^{d} ; W\right)$ when $W \in A P_{p}$.
We need the following Lemma which gives an estimate of the norm of integral operators on $L_{2}\left(\mathbb{T}^{d} ; W\right)$ with nice compactly supported kernels.

Lemma 6. Suppose $S f(\xi)=\int_{\mathbb{T}^{d}} S(\xi, \eta) f(\eta) d \eta$ is an integral operator with a scalar kernel $S(\xi, \eta)$ that satisfies $|S(\xi, \eta)| \leq$ $\alpha|R|^{-1} \chi_{R \times R}$ for some bounded rectangle $R \subset \mathbb{R}^{d}$. For $1<p<$ $\infty$, there exists a constant $C_{d}$ independent of the particular choice of $S$ such that the norm of $S$ on $L_{p}\left(\mathbb{T}^{d} ; W\right)$ is at most $C_{d} \cdot \alpha \cdot A_{W}(R, p)$, with $A_{W}(R, p)$ given by $(8)$. Moreover, the kernel $\alpha|R|^{-1} \chi_{R} \times \chi_{R}$ induces an operator with norm at least $C_{d}^{-1} \cdot \alpha \cdot A_{W}(R, p)$ on $L_{p}\left(\mathbb{T}^{d} ; W\right)$.

The proof of Lemma 6 for nonproduct $A_{p}$-weights can be found in Goldberg [10]. We leave the straightforward adaptation of the proof in [10] to the product case for the reader.

We can now give a proof of Proposition 7. For $K \in \mathbb{N}$, we let $\mathscr{P}_{K}=\operatorname{span}\left\{e^{2 \pi i k \cdot}: k \in \mathbb{Z} ;|k| \leq K\right\}$.

Proposition 7. Let $W: \mathbb{T}^{d} \rightarrow M$ be a periodic matrix weight, and let $\left\{K_{n}\right\}_{n \geq 1}$ be a sequence of real-valued trigonometric convolution kernels defined on $\mathbb{T}$. Assume there exist constants c, $C$ such that $K_{n} \in \mathscr{P}_{c \cdot n}$, with $C^{-1} n \leq\left\|K_{n}\right\|_{\infty}=K_{n}(0) \leq$ $C n$, for $n \in \mathbb{N}$. Suppose that the corresponding product kernels

$$
K_{\mathbf{N}}(\xi)=\prod_{j=1}^{d} K_{N_{j}}\left(\xi_{j}\right), \quad \mathbf{N} \in \mathbb{N}_{0}^{d}
$$

induce a uniformly bounded family $\left\{T_{K_{N}}\right\}$ of convolution operators on $L_{p}\left(\mathbb{T}^{d} ; W\right)$. Then $W \in P A_{p}$.

Proof. We have to estimate $A_{W}(R, p)$ for an arbitrary rectangle $R \in \mathscr{R}(d)$. The idea is to form a suitable product kernel $K_{\mathrm{N}}$ that is "large" on $R$ in the sense that the corresponding operator can be well approximated by an integral operator of the type considered in Lemma 6.

By assumption, the kernel $K_{n} \in \mathscr{P}_{c \cdot n}$ is real and $\left\|K_{n}\right\|_{\infty}=$ $K_{n}(0) \leq C n$, so by Bernstein's inequality, $\left\|K_{n}^{\prime}\right\|_{\infty} \leq c C n^{2}$. We can thus find an integer $M$ (independent of $n$ ) such that for $t \in[-1 / M n, 1 / M n]$ we have $K_{n}(t) \geq\left(1-\left(1 / 2 C_{d}^{2}\right)\right)^{1 / d}\left\|K_{n}\right\|_{\infty}$, where $C_{d}$ is the constant from Lemma 6 .

Let a rectangle $R=I_{1} \times I_{2} \times \cdots \times I_{d}$ be given. For $j=$ $1,2, \ldots, d$, with $\left|I_{j}\right|>1 / 2 M$, we define $N_{j}=0$ and replace $I_{j}$ with $[-1 / 2,1 / 2)$ and obtain a possibly larger rectangle $\widetilde{R}$. By Lemma 4(b), there exists a universal constant $b$ such that $A_{W}(R, p) \leq b A_{W}(\widetilde{R}, p)$ since $|\widetilde{R}| \leq(2 M)^{d}|R|$. Next, for each $j=1,2, \ldots, d$ with $\left|I_{j}\right| \leq 1 / 2 M$, we choose an integer $N_{j} \geq 1$ such that

$$
\frac{1}{4 M} \cdot \frac{1}{N_{j}} \leq\left|I_{j}\right| \leq \frac{1}{2 M} \cdot \frac{1}{N_{j}} .
$$


Notice that for $t, u \in I_{j}$, we have $t-u \in I_{j}-I_{j} \subset$ $\left[-1 / M N_{j}, 1 / M N_{j}\right]$ so

$$
K_{N_{j}}(t-u) \geq\left(1-\frac{1}{2 C_{d}^{2}}\right)^{1 / d}\left\|K_{N_{j}}\right\|_{\infty}
$$

For notational convenience we put $K_{0}:=1$ and form the product kernel

$$
K_{\mathbf{N}}(\xi)=\prod_{j=1}^{d} K_{N_{j}}\left(\xi_{j}\right)
$$

The plan of attack is to use the simple fact that $f \rightarrow$ $\chi_{\widetilde{R}} T_{K_{\mathrm{N}}}\left(\chi_{\widetilde{R}} f\right)$ is uniformly bounded in both $\widetilde{R}$ and $\mathbf{N} \in \mathbb{N}_{0}^{d}$. We notice that $f \rightarrow \chi_{\widetilde{R}} T_{K_{\mathrm{N}}}\left(\chi_{\widetilde{R}} f\right)$ has integral kernel

$$
S_{2}(\xi, \eta):=\chi_{\widetilde{R}}(\eta) \chi_{\widetilde{R}}(\xi) K_{\mathbf{N}}(\eta-\xi) .
$$

We wish to estimate the operator norm of $S_{2}$ from below. For that purpose we first consider the operator with kernel

$$
\begin{aligned}
S(\xi, \eta) & :=S_{1}(\xi, \eta)-S_{2}(\xi, \eta) \\
& :=\left\|K_{\mathbf{N}}\right\|_{\infty} \chi_{\widetilde{R}}(\xi) \chi_{\widetilde{R}}(\eta)-\chi_{\widetilde{R}}(\xi) \chi_{\widetilde{R}}(\eta) K_{\mathbf{N}}(\xi-\eta) .
\end{aligned}
$$

Notice that estimate (22), together with the fact that $\left\|K_{\mathrm{N}}\right\|_{\infty}=$ $\prod_{j=1}^{d} K_{N_{j}}(0)$, implies the following size estimate

$$
\begin{aligned}
& |S(\xi, \eta)| \\
& \quad=\left|\left\|K_{\mathrm{N}}\right\|_{\infty} \chi_{\widetilde{R}}(\xi) \chi_{\widetilde{R}}(\eta)-\chi_{\widetilde{R}}(\xi) \chi_{\widetilde{R}}(\eta) K_{\mathbf{N}}(\xi-\eta)\right| \\
& \quad \leq \frac{\left\|K_{\mathrm{N}}\right\|_{\infty}}{2 C_{d}^{2}} \chi_{\widetilde{R}}(\xi) \chi_{\widetilde{R}}(\eta) \\
& \quad=\frac{|\widetilde{R}| \cdot\left\|K_{\mathrm{N}}\right\|_{\infty}}{2 C_{d}^{2}}|\widetilde{R}|^{-1} \chi_{\widetilde{R}}(\xi) \chi_{\widetilde{R}}(\eta) .
\end{aligned}
$$

According to Lemma 6, the kernel $S$ induces an operator of norm at most $(1 / 2) C_{d}^{-1}|\widetilde{R}| \cdot\left\|K_{\mathrm{N}}\right\|_{\infty} A_{W}(\widetilde{R}, p)$ on $L_{p}\left(\mathbb{T}^{d} ; W\right)$. At the same time, Lemma 6 shows that the operator with kernel $S_{1}(\xi, \eta)=|\widetilde{R}|\left\|K_{\mathrm{N}}\right\|_{\infty} \cdot|\widetilde{R}|^{-1} \chi_{\widetilde{R}}(\xi) \chi_{\widetilde{R}}(\eta)$ has norm at least $C_{d}^{-1}|\widetilde{R}| \cdot\left\|K_{\mathrm{N}}\right\|_{\infty} A_{W}(\widetilde{R}, p)$ on $L_{p}\left(\mathbb{T}^{d} ; W\right)$. The triangle inequality for operator norms now implies that

$$
\begin{aligned}
& \frac{1}{2 C_{d}}|\widetilde{R}| \cdot\left\|D_{\mathrm{N}}\right\|_{\infty} M(\widetilde{R}, W) \\
& \quad \geq\left\|S_{1}-S_{2}\right\| \geq\left|\left\|S_{1}\right\|-\left\|S_{2}\right\|\right| \\
& \quad \geq C_{d}^{-1}|\widetilde{R}| \cdot\left\|D_{\mathrm{N}}\right\|_{\infty} M(\widetilde{R}, W)-\left\|S_{2}\right\|,
\end{aligned}
$$

so $\left\|S_{2}\right\| \geq(1 / 2) C_{d}^{-1}|\widetilde{R}| \cdot\left\|D_{\mathrm{N}}\right\|_{\infty} A_{W}(\widetilde{R}, p)$. Moreover, by (21), we see that $|\widetilde{R}| \cdot\left\|K_{\mathrm{N}}\right\|_{\infty} \geq C(4 M)^{-d}$, so we may conclude that

$$
\begin{aligned}
A_{W}(R, p) & \leq b A_{W}(\widetilde{R}, p) \\
& \leq 2 b C C_{d}(4 M)^{d}\left\|S_{2}\right\| \\
& =C^{\prime} \sup _{\|f\|_{L_{p}\left(\mathbb{T}^{d} ; W\right)} \| 1}\left\|\chi_{\widetilde{R}} T_{K_{\mathrm{N}}}\left(\chi_{\widetilde{R}} f\right)\right\|_{L_{p}\left(\mathbb{T}^{d} ; W\right)} \\
& \leq C^{\prime} \sup _{\|f\|_{L_{p}\left(\mathbb{T}^{d} ; W\right)}=1}\left\|T_{K_{\mathrm{N}}} f\right\|_{L_{p}\left(\mathbb{T}^{d} ; W\right)} \leq C^{\prime \prime}
\end{aligned}
$$

with constant $C^{\prime \prime}$ independent of $R$. We may finally conclude that $W \in P A_{p}(d)$.

\section{Summation of Multiple Trigonometric Series}

This section contains applications of the results of Section 2 to convolution operators induced by rectangular Dirichlet, Fejér, and Jackson kernels. The Dirichlet kernels correspond to standard rectangular trigonometric summation while the Fejér kernels generate the corresponding Cesàro means. The Jackson kernels are (normalized) squares of the Fejér kernels, and they induce the well-known Jackson approximation by trigonometric polynomials.

We begin by studying the univariate Dirichlet kernel. The Hilbert transform $H$ is defined on $L_{p}(\mathbb{T}), 1<p<\infty$, by

$$
H(f)(x):=\text { p.v. } \int_{\mathbb{V}} f(t) \cot (\pi(x-t)) d t .
$$

We lift $H$ to a linear operator on $L_{p}(\mathbb{T} ; W)$, for any matrix weight $W: \mathbb{T} \rightarrow \mathscr{M}$, by letting it act coordinatewise.

Treil and Volberg completely characterized when the Hilbert transform $H$ is bounded in the matrix case on $\mathbb{T}$ when $p=2$; see [11]. Later, Nazarov and Treull introduced in a new "Bellman function" method [6] to extend the theory to $1<p<\infty$. Volberg presented a different solution to the matrix weighted $L_{p}$ boundedness of the Hilbert transform via Littlewood-Paley theory [7]. The fundamental result is the following.

Theorem 8 (see $[6,7,11])$. Let $W: \mathbb{T} \rightarrow \mathscr{M}$ be a matrix weight. Suppose $1<p<\infty$. Then the Hilbert transform is bounded on $L_{p}(\mathbb{T} ; W)$ if and only if $W \in A_{p}(\mathbb{T})$. by

We recall that the univariate Dirichlet kernel $D_{N}$ is given

$$
D_{N}(t)=\frac{\sin 2 \pi(N+1 / 2) t}{\sin \pi t}, \quad N \geq 1,
$$


and for $f \in L_{p}(\mathbb{T})$ we define the associated partial sum operators,

$$
\begin{aligned}
S_{N}(f): & =\sum_{k=-N}^{N} \hat{f}(k) e^{2 \pi i k .} \\
& =f * D_{N}:=\int_{\mathbb{T}} f(t) D_{N}(\cdot-t) d t .
\end{aligned}
$$

We have the following lemma which follows easily from Theorem 8 .

Lemma 9. Let $W: \mathbb{T} \rightarrow \mathscr{M}$ be a matrix weight in $A_{p}(\mathbb{T})$. Then the partial sum operators $f \rightarrow f * D_{N}$ are uniformly bounded on $L_{p}(\mathbb{T} ; W)$.

Proof. We let $P_{+}=(1 / 2)\left(I+i H+S_{0}\right)$ denote the Riesz projection onto $H^{p}$ for $f \in L_{p}(\mathbb{T} ; W)$, where $S_{0} f:=$ $\int_{\mathbb{T}} f(y) d y$ is the 0 -order partial sum operator. It follows that $P_{+}$is bounded on $L_{p}(\mathbb{T} ; W)$ since $H$ is bounded according to Theorem 8, and $S_{0}$ is bounded according to [12, Lemma 1.5]. Notice that $f \rightarrow f e^{2 \pi i M \cdot}$ is a norm preserving operator on $L_{p}(\mathbb{T} ; W)$, just as in the scalar case. Then we observe that

$$
f * D_{N}=e^{-2 \pi i N \cdot} P_{+}\left(e^{2 \pi i N \cdot} f\right)-e^{2 \pi i(N+1) \cdot} P_{+}\left(e^{-2 \pi i(N+1) \cdot} f\right),
$$

and the result follows.

For $\mathbf{N}=\left(N_{1}, \ldots, N_{d}\right) \in \mathbb{N}_{0}^{d}$, we form the product kernel $D_{\mathbf{N}}(\xi):=\prod_{j=1}^{d} D_{N_{j}}\left(\xi_{j}\right)$. One has

$$
S_{\mathbf{N}}(\mathbf{f}):=\int_{\mathbb{T}^{d}} \mathbf{f}(t) D_{\mathbf{N}}(\cdot-t) d t .
$$

We notice that $D_{N} \in \mathscr{P}_{N}$ and $D_{N}(0)=\left\|D_{N}\right\|_{\infty}=2 N+1$, so the following corollary follows directly from Propositions 5 and 7 and Lemma 9.

Corollary 10. Let $W: \mathbb{T}^{d} \rightarrow M$ be a matrix weight. For $1<$ $p<\infty$, the operators $\left\{S_{\mathbf{N}}: \mathbf{N} \in \mathbb{N}^{d}\right\}$ are uniformly bounded on $L_{p}\left(\mathbb{T}^{d} ; W\right)$ if and only if $W \in A P_{p}$.

Remark 11. It is easy to verify that vectors of trigonometric polynomials are dense in $L_{p}\left(\mathbb{T}^{d} ; W\right), 1<p<\infty$, whenever $W$ is a matrix weight (since $W \in L_{1}$ so each entry in $W$ is in $\left.L_{1}(\mathbb{T})\right)$. It therefore follows by standard techniques that the family $\left\{S_{\mathbf{N}}: \mathbf{N} \in \mathbb{N}^{d}\right\}$ is uniformly bounded on $L_{p}\left(\mathbb{T}^{d} ; W\right)$ if and only if $\left\|\mathbf{f}-S_{\mathbf{N}}(\mathbf{f})\right\|_{L_{p}(\mathbb{T} ; W)} \rightarrow 0$, as $\min _{j} N_{j} \rightarrow+\infty$, for all $\mathbf{f} \in L_{p}(\mathbb{T} ; W)$.

Corollary 10 relies on basic localization properties of the Dirichlet kernel. However, many well-known summation kernels share the necessary properties needed to apply Propositions 5 and 7. Let us illustrate this fact by considering two specific examples.
The rectangular Cesàro summation is given by

$$
\begin{aligned}
\sigma_{\mathbf{N}}(\mathbf{f}) & =\frac{1}{\left(N_{1}+1\right) \cdots\left(N_{d}+1\right)} \sum_{k_{1}=0, \ldots, k_{d}=0}^{N_{1}, \ldots, N_{d}} S_{\left(k_{1}, k_{2}, \ldots, k_{d}\right)}(\mathbf{f}) \\
& =\int_{\mathbb{T}^{d}} \mathbf{f}(t) F_{\mathbf{N}}(\cdot-t) d t
\end{aligned}
$$

with the product Féjer kernel given by $F_{\mathbf{N}}(\xi)=\prod_{j=1}^{d} F_{N_{j}}\left(\xi_{j}\right)$, where the scalar Féjer kernel is defined by

$$
F_{n}(t)=\frac{1}{n}\left(\frac{\sin (n \pi t)}{\sin (\pi t)}\right)^{2} .
$$

Notice that $F_{b} \in \mathscr{P}_{n}$ and $F_{n}(0)=\left\|F_{n}\right\|_{\infty}=n$. The scalar Jackson kernel is the normalized square of the Féjer kernel and given by

$$
J_{n}(t)=\frac{3}{n\left(2 n^{2}+1\right)}\left(\frac{\sin (n \pi t)}{\sin (\pi t)}\right)^{4} .
$$

The corresponding product kernel is $J_{\mathbf{N}}(\xi)=\prod_{j=1}^{d} J_{N_{j}}\left(\xi_{j}\right)$, $\mathbf{N} \in \mathbb{N}_{0}^{d}$, and the rectangular Jackson summation operator is given by $\mathscr{J}_{\mathbf{N}}(f):=f * J_{\mathbf{N}}$. Notice that $J_{n} \in \mathscr{P}_{2 n}$ and $J_{n}(0)=$ $\left\|J_{n}\right\|_{\infty}=n$.

We now conclude by stating the main result, which summarizes the results obtained in the present paper. The theorem shows that uniform boundedness of the rectangular operators $S_{\mathbf{N}}, \sigma_{\mathbf{N}}$, and $\mathscr{J}_{\mathbf{N}}$ on $L_{p}\left(\mathbb{T}^{d} ; w\right)$ is equivalent to the condition $W \in A P_{p}$.

Theorem 12. Let $W: \mathbb{T}^{d} \rightarrow M$ be a matrix weight. For $1<$ $p<\infty$, the following conditions are equivalent:

(i) $W \in A P_{p}$;

(ii) the operators $\left\{S_{\mathbf{N}}: \mathbf{N} \in \mathbb{N}_{0}^{d}\right\}$ are uniformly bounded on $L_{p}\left(\mathbb{T}^{d} ; W\right) ;$

(iii) the operators $\left\{\sigma_{\mathbf{N}}: \mathbf{N} \in \mathbb{N}_{0}^{d}\right\}$ are uniformly bounded on $L_{p}\left(\mathbb{T}^{d} ; W\right) ;$

(iv) the operators $\left\{\mathscr{F}_{\mathbf{N}}: \mathbf{N} \in \mathbb{N}_{0}^{d}\right\}$ are uniformly bounded on $L_{p}\left(\mathbb{T}^{d} ; W\right)$.

Proof. We first notice that each of the univariate kernels $D_{n}, F_{n}$, and $J_{n}$ satisfies the hypothesis of Proposition 7, so (ii), (iii), and (iv) each implies that $W \in A P_{p}$. Now, suppose that $W \in A P_{p}$. Then (ii) holds by Corollary 10 . To conclude, we just need to recall that Cesàro and Jackson summations are both regular summation methods, so (ii) implies both (iii) and (iv).

\section{References}

[1] L. Grafakos, Classical Fourier Analysis, vol. 249 of Graduate Texts in Mathematics, Springer, New York, NY, USA, 2nd edition, 2008. 
[2] M. Nielsen, "On stability of finitely generated shift-invariant systems," The Journal of Fourier Analysis and Applications, vol. 16, no. 6, pp. 901-920, 2010.

[3] B. Muckenhoupt, "Weighted norm inequalities for the Hardy maximal function," Transactions of the American Mathematical Society, vol. 165, pp. 207-226, 1972.

[4] R. Hunt, B. Muckenhoupt, and R. Wheeden, "Weighted norm inequalities for the conjugate function and Hilbert transform," Transactions of the American Mathematical Society, vol. 176, pp. 227-251, 1973.

[5] F. Nazarov, S. Treil, and A. Volberg, "The Bellman functions and two-weight inequalities for Haar multipliers," Journal of the American Mathematical Society, vol. 12, no. 4, pp. 909-928, 1999.

[6] F. L. Nazarov and S. R. Treıll/, "The hunt for a Bellman function: applications to estimates for singular integral operators and to other classical problems of harmonic analysis," Algebra i Analiz, vol. 8, no. 5, pp. 32-162, 1996.

[7] A. Volberg, "Matrix $A_{p}$ weights via S-functions," Journal of the American Mathematical Society, vol. 10, no. 2, pp. 445-466, 1997.

[8] S. Roudenko, "Matrix-weighted Besov spaces," Transactions of the American Mathematical Society, vol. 355, no. 1, pp. 273-314, 2003.

[9] S. Y.A. Chang and R. Fefferman, "Some recent developments in Fourier analysis and $H^{p}$-theory on product domains," Bulletin of the American Mathematical Society, vol. 12, no. 1, pp. 1-43, 1985.

[10] M. Goldberg, "Matrix $A_{p}$ weights via maximal functions," Pacific Journal of Mathematics, vol. 211, no. 2, pp. 201-220, 2003.

[11] S. Treil and A. Volberg, "Wavelets and the angle between past and future," Journal of Functional Analysis, vol. 143, no. 2, pp. 269-308, 1997.

[12] S. Treil and A. Volberg, "Continuous frame decomposition and a vector Hunt-Muckenhoupt-Wheeden theorem," Arkiv för Matematik, vol. 35, no. 2, pp. 363-386, 1997. 


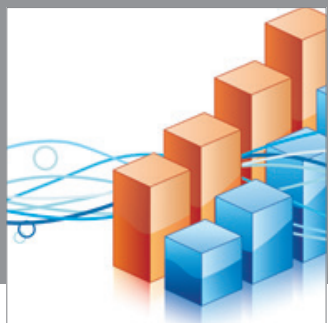

Advances in

Operations Research

mansans

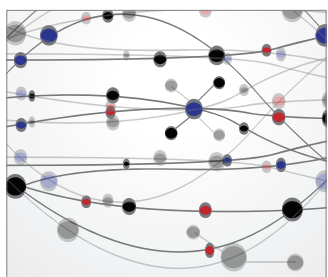

The Scientific World Journal
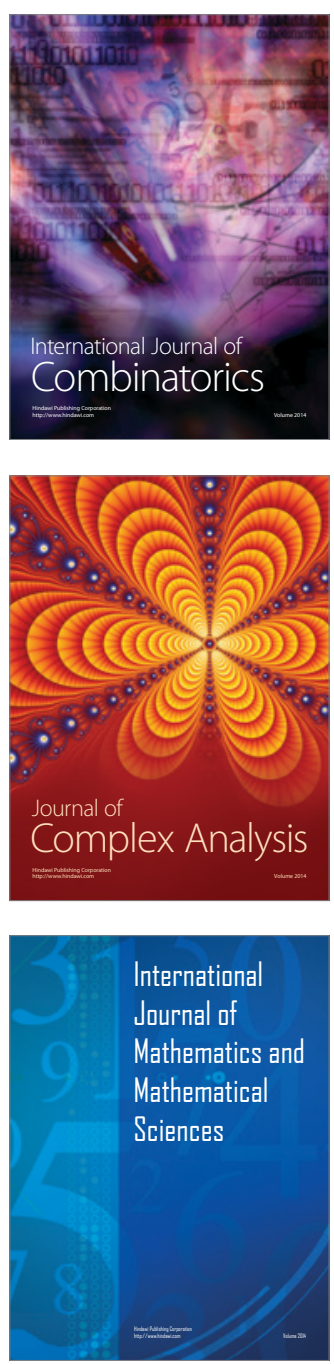
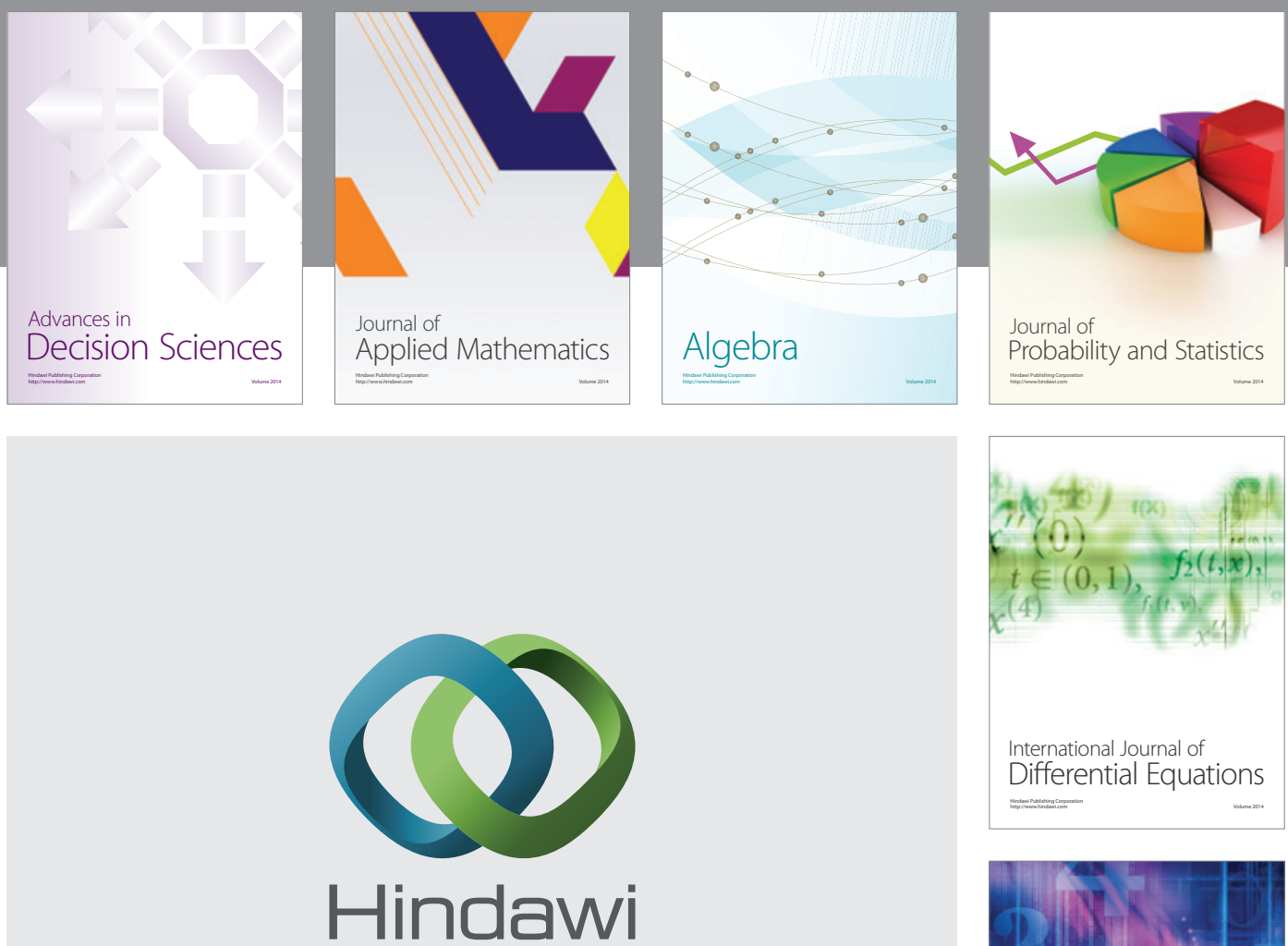

Submit your manuscripts at http://www.hindawi.com
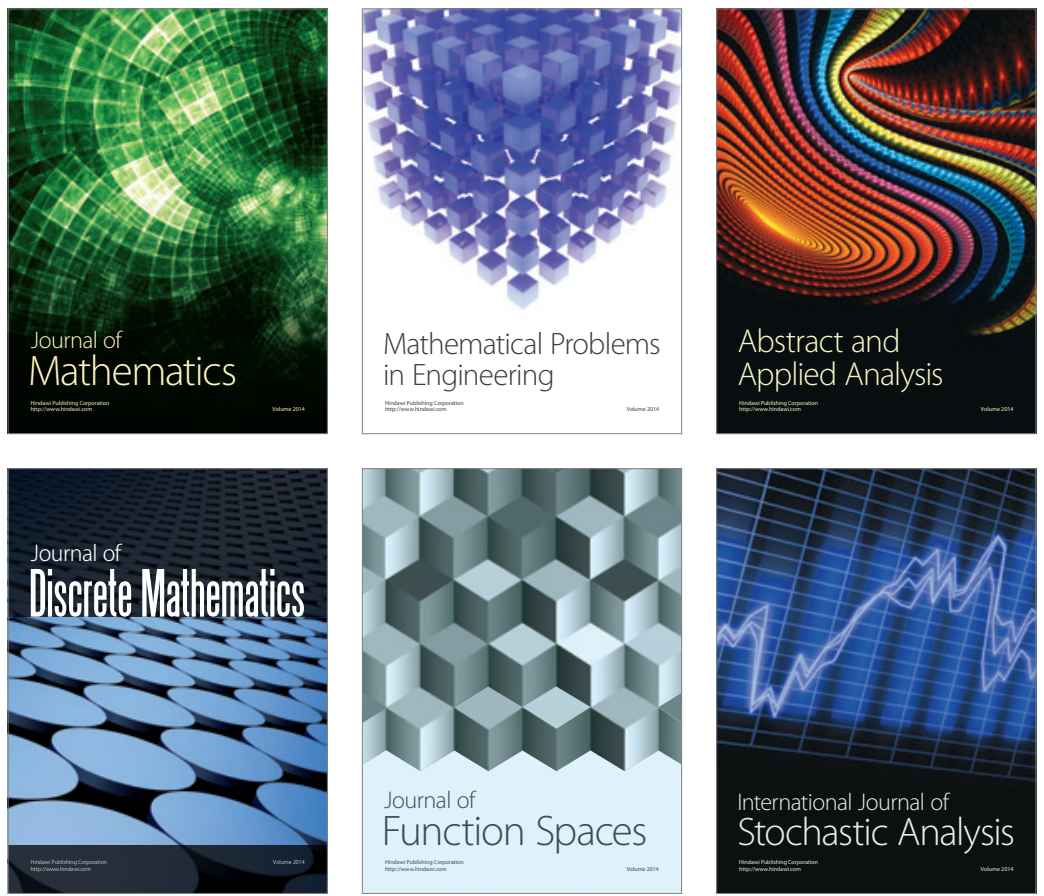

Journal of

Function Spaces

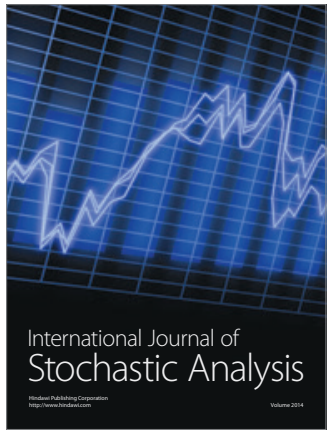

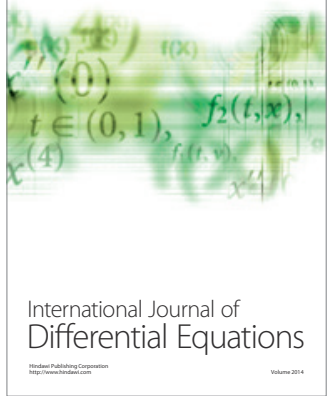
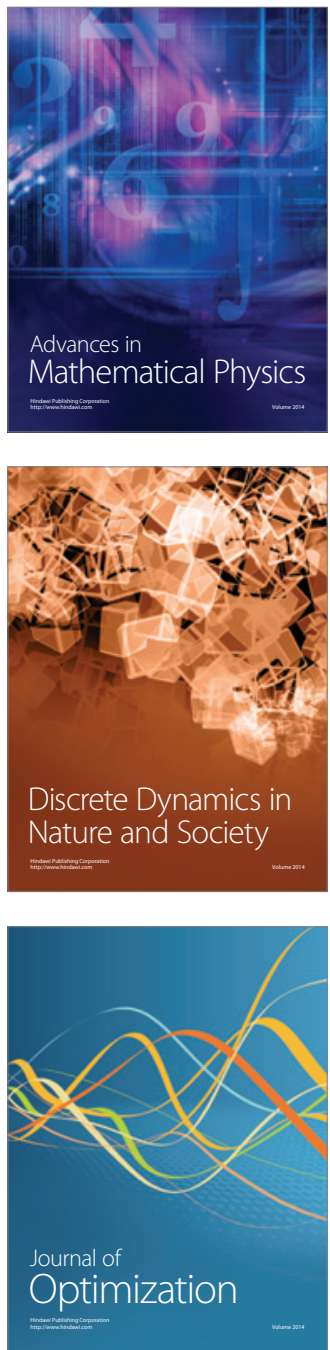